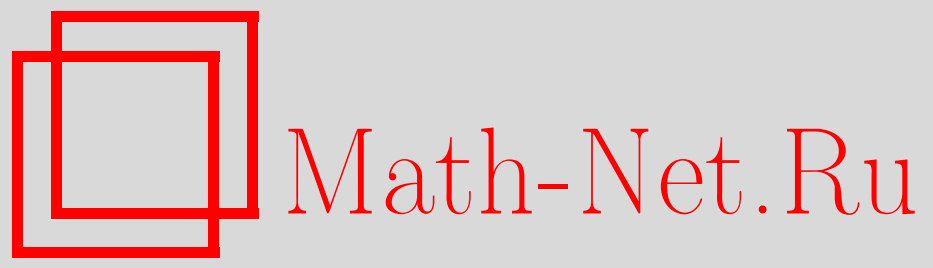

Обцероссийский математический портал

С. Ю. Доброхотов, Е. С. Семенов, Б. Тироцци, Цепочки Гюгонио-Маслова для системы уравнений мелкой воды с учетом энергетического обмена, Матем. заметки, 2005, том 78, выпуск 5, 796-799

DOI: https://doi.org/10.4213/mzm2643

Использование Общероссийского математического портала Math-Net.Ru подразумевает, что вы прочитали и согласны с пользовательским соглашением http://www.mathnet.ru/rus/agreement

Параметры загрузки:

IP : 54.89 .56 .158 
26 апреля 2023 г., 15:49:57

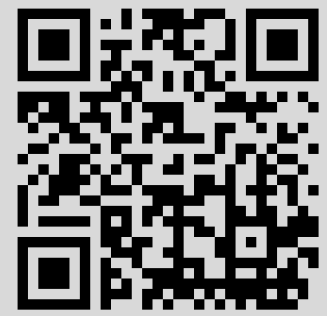




\section{ЦЕПОЧКИ ГЮГОНИО-МАСЛОВА ДЛЯ СИСТЕМЫ УРАВНЕНИЙ МЕЛКОЙ ВОДЫ С УЧЕТОМ ЭНЕРГЕТИЧЕСКОГО ОБМЕНА}

\section{С. Ю. Доброхотов, Е. С. Семёнов, Б. Тироцци}

Особые решения системы уравнений мелкой воды со структурой “типа квадратного корня" из квадратичной формы были введены в работе [1]. Таким решениям сопоставляется цепочка Гюгонио-Маслова - бесконечная цепочка обыкновенных дифференциальных уравнений, по существу определяющих их динамику. Эти цепочки обладают многими интересными свойствами (см. [2]-[7]). Цель настоящей заметки состоит в описании цепочки Гюгонио-Маслова для более сложной системы уравнений, описьвающих динамику двумерных вихрей в атмосфере с учетом энергетического обмена между атмосферой и океаном [8]. После обезразмеривания эта система выглядит следующим образом:

$$
\begin{gathered}
\frac{\partial \mathbf{u}}{\partial t}+\mathbf{u} \cdot \nabla \mathbf{u}-\omega \mathbf{T u}+\nabla \eta+(\eta+\delta) \nabla \Theta=0 \\
\frac{\partial \eta}{\partial t}+\nabla \cdot\left(\eta \mathbf{u}+\delta \operatorname{curl}_{3} \mathbf{u}-\eta k \Theta\right)=0 \\
\frac{\partial \Theta}{\partial t}+\mathbf{u} \cdot \nabla \Theta+k \Theta=0
\end{gathered}
$$

Неизвестными в этой системе являются зависящие от координат $x={ }^{t}\left(x_{1}, x_{2}\right)$ и времени $t$ двумерная вектор-функция скорости $\mathbf{u}(x, t)$ и скалярные функции $\eta(x, t)$ (геопотенциал) и $\Theta(x, t)($ соответствует разнице температуры атмосферы и температуры поверхности океана); $\nabla={ }^{t}\left(\partial / \partial x_{1}, \partial / \partial x_{2}\right)$, $\mathbf{T}=\left(\begin{array}{cc}0 & 1 \\ -1 & 0\end{array}\right)$ - матрица поворота на угол $\pi / 2, \operatorname{curl}_{3} \mathbf{u}=\partial \mathbf{u}_{2} / \partial x_{1}-\partial \mathbf{u}_{1} / \partial x_{2}, \omega=\widetilde{\omega}+\beta x_{2}-$ удвоенная частота Кориолиса на $\beta$-плоскости, $\omega_{0}, \beta, \delta, k$ - физические константы, в частности, константа $\delta$ характеризует приток массы из пограничного слоя атмосферы - слоя Экмана [8], [9] (эффект наличия вязкости и условия Дирихле для скорости ветра на границе атмосферы и океана в начальной трехмерной модели). После сведения этой задачи к двумерной остается слагаемое $\delta \operatorname{curl}_{3} \mathbf{u}$ в уравнении (2); константа $k$ характеризует нагрев вихря (см. [8]).

Работа выполнена при поддержке Российского фонда фундаментальных исследований, грант № 02-01-00850. 
Особые решения со структурой “типа квадратного корня” из квадратичной формы имеют вид

$$
\begin{array}{ll}
\mathbf{u}=u(x, t)+\widetilde{u}(x, t), & \widetilde{u}(x, t)=U(x, t) \sqrt{S(x, t)}, \\
\eta=\rho(x, t)+\widetilde{\rho}(x, t), & \widetilde{\rho}(x, t)=R(x, t) \sqrt{S(x, t)}, \\
\Theta=\theta(x, t)+\widetilde{\theta}(x, t), & \widetilde{\theta}(x, t)=N(x, t) \sqrt{S(x, t)} .
\end{array}
$$

Здесь гладкие функции $u, \rho, \theta$ определяют “фон”, $U, N, R$ - “амплитуду", $S$ - “фазу”. Относительно функции $S$ предполагается (cp. [6]), что

(i) $S \geqslant 0, S$ обращается в нуль только на траектории (особенности)

$$
\Gamma=\left\{x=X(t) \equiv\left(X_{1}(t), X_{2}(t)\right)\right\}
$$

и матрица вторых производных $\left\|\frac{\partial^{2} S}{\partial x_{i} x_{j}}(X(t), t)\right\|$ строго положительна и имеет различные собственные значения.

При $\delta=0, k=0$ и $\Theta=0$ система уравнений (1)-(3) переходит в систему уравнений мелкой воды на $\beta$-плоскости для неизвестных $\mathbf{u}, \eta$. Смысл решений вида (4)-(6) для такой системы, их различные свойства и приложения к теории движения тропических циклонов в атмосфере подробно обсуждался в работах [2]-[7]. В работе [3] было доказано, что решения (4), (5) являются единственными возможньми структурно устойчивыми и самоподобными слабыми особыми решениями уравнений мелкой воды на $\beta$-плоскости при условии общего положения начальных условий. Цепочка Гюгонио-Маслова, отвечающая решениям (4)-(6), - это незамкнутая бесконечная система обыкновенных дифференциальных уравнений, связьвающая соответствующие тейлоровские коэфофициенты функций $u_{1}, u_{2}, \rho, \theta, U_{1}, U_{2}, R, N$ и $S$. Для особых решений типа ударных волн первые из уравнений аналогичной цепочки - хорошо известные условия Гюгонио, остальные могут быть рассмотрены как поправки к ним. В [1]-[6] показано, что в качестве аналогов условий Гюгонио для решений типа (4), (5) системы уравнений мелкой воды появляются условие "вмороженности" центра особенности в поле скоростей $u(x, t)$ :

$$
\dot{X}=\left.\left.u\right|_{\Gamma} \stackrel{\text { def }}{=} V \equiv \mathbf{u}\right|_{\Gamma}
$$

и условия Коши-Римана для комплексной скорости $u_{1}+i u_{2}$ на траектории особенности:

$$
\frac{\partial u_{1}}{\partial x_{1}}=\left.\frac{\partial u_{2}}{\partial x_{2}}\right|_{\Gamma},\left.\quad \frac{\partial u_{1}}{\partial x_{2}}\right|_{\Gamma}=-\left.\frac{\partial u_{2}}{\partial x_{1}}\right|_{\Gamma} .
$$

При этом относительно функций $U, R$ предполагалось, что

(ii) разложение в ряд Тейлора по $x$ функций $U, R$ в окрестности траектории $X(t)$ начинается с минимально возможных степеней $x-X(t)$.

Цель этой заметки - описание цепочек Гюгонио-Маслова и их некоторых свойств для решений (4)-(6) уравнений (1)-(3). Эти условия получаются в результате реализации схемы [6]. Анализ уравнений, порожденных "негладкой частью", приводит к соотношениям, позволяющим выразить фазу $S$ и амплитуды $U, R, N$ через гладкие составляющие решения $u, \rho, \theta$.

Теорема 1. Пусть выполнены условия (i), (ii). Тогда выполнено условие вооруженности (7) и без уменьшения общ,ности мохно считать, что функиия $S$ удовлетворяет уравнению Гамильтона-Якоби

$$
\frac{d}{d t} S=\frac{\partial}{\partial t} S+(u, \nabla) S=0
$$

При этом

1) если $\left.N\right|_{\Gamma} \neq 0$, то функции $\rho$ и $\theta$ удовлетворяют соотношению $\left.(\nabla \rho+(\rho+\delta) \nabla \theta)\right|_{\Gamma}=0$ и траектория $X(t)$ чентра особенности описьвается системой уравнений

$$
\dot{X}=V, \quad \dot{V}=\omega_{0} \mathbf{T} V, \quad \dot{\omega}_{0}=\beta V_{2}, \quad \text { əде } \quad \omega_{0}=\left.\omega\right|_{\Gamma} ;
$$


2) если $\left.N\right|_{\Gamma}=0$, то $\left.R\right|_{\Gamma}=0$ и на траектории особенности $Г$ выполнены условия Коши-Римана для комплексной функиии $w_{1}(x, t)+i w_{2}(x, t)$, әде

$$
{ }^{t}\left(w_{1}, w_{2}\right)=(\mathbf{T} P, \nabla N)(\nabla \rho+(\rho+\delta) \nabla \theta)+2 \operatorname{det}\left\|\frac{\partial^{2} S}{\partial x_{i} x_{j}}(X(t), t)\right\| u ;
$$

3) в случае, когда $\left.N\right|_{\Gamma}=\left.\nabla N\right|_{\Gamma}=0$ с необходимостью справедливо $\left.\nabla \theta\right|_{\Gamma}=0$, выполнень условия Коши-Римана (8), а негладкая часть решения имеет следующую структуру:

$$
\begin{gathered}
\widetilde{u}=\left(\phi(t)\left(\mathbf{T} \nabla S+\frac{\delta}{\rho} \nabla S\right)+O\left(\|x-X(t)\|^{2}\right)\right) \sqrt{S} \\
R=O\left(|x-X(t)|^{2}\right), \quad N=O\left(|x-X(t)|^{2}\right), \\
R+(\rho+\delta) N=-\frac{2}{3} \phi(t)(\operatorname{curl} u+\omega) S+O\left(\|x-X(t)\|^{3}\right), \\
\dot{\phi}=\delta C \phi, \quad C=\left.\frac{\operatorname{curl} u+\omega}{\rho}\right|_{\Gamma} .
\end{gathered}
$$

Случай 1) теоремы 1 не представляет интереса с точки зрения приложений, так как он дает быстрые траектории, отвечающие частоте $\omega_{0}$. Ограничимся рассмотрением случая 3) теоремы 1. Мы также предполагаем, что $C>0$ (см. [6]).

Другие уравнения из цепочки Гюгонио-Маслова получаются приравниванием коэффициентов Тейлора к нулю при одинаковых степенях в уравнениях, порожденных гладкой частью решения. Вычисления удобно проводить в комплексных координатах (см. [7]) $z=x_{1}-X_{1}+i\left(x_{2}-X_{2}\right)$, $\bar{z}=x_{1}-X_{1}-i\left(x_{2}-X_{2}\right)$, используя комплексные производные

$$
\frac{\partial}{\partial z}=\frac{1}{2}\left(\frac{\partial}{\partial x_{1}}-i \frac{\partial}{\partial x_{2}}\right), \quad \frac{\partial}{\partial \bar{z}}=\frac{1}{2}\left(\frac{\partial}{\partial x_{1}}+i \frac{\partial}{\partial x_{2}}\right)
$$

и комплексные функции $X(t)=X_{1}(t)+i X_{2}(t), V(t)=V_{1}(t)+i V_{2}(t), u=u_{1}+i u_{2}$ и т.д. вместо соответствующих двумерных векторных функций. Введем также следующие функции:

$$
\begin{gathered}
\rho_{0}=\left.\rho\right|_{\Gamma}, \quad q=\left.\frac{1}{2} \operatorname{div} u\right|_{\Gamma}, \quad p=-\frac{1}{2} \operatorname{curl}_{3} u, \quad \mu=\frac{1}{\sqrt{\rho_{0} C}} \\
Y=\mu^{3}\left(\left.2 \frac{\partial^{2} u}{\partial z \partial \bar{z}}\right|_{\Gamma}-\frac{\beta}{3}\right), \quad Z=\mu^{3}\left(\left.\frac{\partial^{2} u}{\partial z \partial z}\right|_{\Gamma}+\frac{\beta}{3}\right), \\
U=\left.\mu^{3} \frac{\partial^{2} u}{\partial \bar{z} \partial \bar{z}}\right|_{\Gamma}, \quad W=-\left.\mu^{3} \rho_{0} 2 i \frac{\partial \Pi}{\partial \bar{z}}\right|_{\Gamma}, \\
\tau=\frac{\partial^{2} \theta /\left.\partial z \partial \bar{z}\right|_{\Gamma}}{\rho_{0} C}, \quad \xi=\frac{\partial^{2} \theta /\left.\partial z \partial z\right|_{\Gamma}}{\rho_{0} C}, \quad r=\left.\mu^{4} \frac{\partial^{2} \rho}{\partial \bar{z} \partial z}\right|_{\Gamma}+\frac{\tau}{C} .
\end{gathered}
$$

Теорема 2. Первые 11 уравнений чепочки Гюгонио-Маслова имеют вид

$$
\begin{gathered}
\dot{X}-V=0 \\
\dot{V}+i \omega_{0} V-\frac{i}{C \mu^{3}}(Y+W-2 \bar{Z})=0, \\
\dot{Y}-i\left(p-\omega_{0}\right) Y+\frac{i}{3} \beta\left(2 p+\omega_{0}\right) \mu^{3}=\frac{1}{2} \gamma \beta \mu^{3}+E_{1}+f_{1}, \\
\dot{Z}-i\left(3 p-\omega_{0}\right) Z-\frac{i}{3} \omega_{0} \beta \mu^{3}=-\frac{1}{2} \gamma \beta \mu^{3}+E_{2}+f_{2}, \\
\dot{U}-i\left(p+\omega_{0}\right) U=E_{3}+f_{3},
\end{gathered}
$$




$$
\begin{gathered}
\dot{W}+i p W=-2 C \delta p \mu^{2} W-C \delta \omega_{0} \mu^{2}(Y-2 \bar{Z})-\delta \beta \mu^{3} C+E_{4}, \\
\ddot{\mu}+\frac{\omega_{0}^{2}+2 \beta \operatorname{Re} V}{4} \mu-\mu^{-3}\left(\frac{1}{4}-r\right)=-2 \mu^{-1} \delta \tau, \\
\dot{r}+\frac{1}{\mu^{2} C} \operatorname{Im}((Z+\bar{Y}) W+3 Y Z)=k \theta_{0} r+2 p \delta \mu^{2} \tau, \\
\dot{C}=-\gamma C, \quad \dot{\omega}_{0}-\beta \operatorname{Im} V=0, \\
\dot{\theta}_{0}+k \theta_{0}=0, \quad \dot{\tau}+k \tau=0, \quad \dot{\xi}-2 i p \xi+k \xi=0 .
\end{gathered}
$$

Здесь неизвестными являются комплексные функиии $X(t), V(t), Y(t), Z(t), W(t), U(t) u$ вещ, ественнье функчии $\omega_{0}(t), C(t), r(t), \theta(t), \mu(t)$;

$$
\begin{gathered}
E_{1}=\frac{2 i}{C \mu^{2}}((Y+W-2 \bar{Z}) \tau-(\bar{Y}+\bar{W}-2 Z) \bar{\xi}), \quad E_{2}=-\frac{2 i}{C \mu^{2}}(\bar{Y}+\bar{W}-2 Z) \tau \\
E_{3}=\frac{2 i}{C \mu^{2}}(Y+W-2 \bar{Z}) \bar{\xi}, \quad E_{4}=\frac{2 i}{C \mu^{2}}((Y+W-2 \bar{Z}) \tau+(\bar{Y}+\bar{W}-2 Z) \bar{\xi}) \\
\gamma=\frac{\delta 2 p}{\rho_{0}}+k \theta_{0}, \quad p=\frac{\omega_{0}-\rho_{0} C}{2}, \quad \rho_{0}=\frac{1}{\mu^{2} C}
\end{gathered}
$$

$f_{1}, f_{2}, f_{3}$ зависят от коэффичиентов Тейлора функиий и, $\rho$ третвего порядка.

Для моделирования движения тропических циклонов мы обрьваем цепочку (11)-(20), предполагая $f_{1}=f_{2}=f_{3}=0$.

Оборванная цепочка Гюгонио-Маслова для уравнений мелкой воды без учета энергетического обмена получается из уравнений (11)-(19) после приравнивания к нулю их правых частей.

\section{СПИСОК ЦИТИРОВАННОЙ ЛИТЕРАТУРЫ}

1. Маслов В. П. // УМН. 1980. Т. 35. № 2. С. 252-253. 2. Dobrokhotov S. Yu. // Russ. J. Math. Phys. 1999. V. 6. № 2. P. 137-173; № 3. P. 282-313. 3. Dobrokhotov S. Yu., Pankrashkin K. V., Semenov E. S. // Russ. J. Math. Phys. 2001. V. 8. №1. P. 25-54. 4. Семенов Е. С. // Матем. заметки. 2002. Т. 71. №6. С. 902-913. 5. Доброхотов С. Ю., Тироцци Б. // Докл. РАН. 2002. Т. 384. №6. С. 741-746. 6. Доброхотов С., Семенов Е., Тироцци Б. // Современная математика. Фундаментальные направления. 2003. Т. 2. С. 5-44. 7. Доброхотов С. Ю., Семенов Е. С., Тироцци Б. // ТМФ. 2004. Т. 139. № 1. С. 500-512. 8. Должанский Ф. В., Крымов В.А., Манин Д. Ю. // Успехи физ. наук. 1990. Т. 160. № 7. С. 1-47. 9. Педлоски Дж. Геофизическая гидродинамика. М.: Мир, 1984.

(С. Ю. Доброхотов, Е. С. Семёнов) Институт проблем механики РАН 